\title{
The development of a mouse model to explore resistance and susceptibility to early Ascaris suum infection
}

\author{
R. LEWIS ${ }^{1 *}$, J. M. BEHNKE ${ }^{2}$, P. STAFFORD ${ }^{1}$ and C. V. HOLLAND ${ }^{1}$ \\ ${ }^{1}$ School of Natural Sciences, Department of Zoology, Trinity College, University of Dublin, Dublin 2, Ireland \\ ${ }^{2}$ School of Biology, University of Nottingham, University Park, Nottingham NG7 2RD, UK
}

(Received 18 Fanuary 2005; revised 8 April, 8 Fuly and 10 August 2005; accepted 10 August 2005 ; first published online 6 October 2005)

\author{
S U M MAR Y
}

Ascaris suum and Ascaris lumbricoides exhibit an over-dispersed frequency distribution in their host populations in both the adult and larval stages. The impact of host factors on this observed distribution is still poorly understood and difficult to investigate in the natural host populations. The use of a mouse model has been supported by the observations that the larval migratory pattern, in this host, mimics the pattern observed in the pig. We explored the extrinsic factors that might affect the quantitative recovery of larvae during this migration in order to standardize a model system facilitating accurate future assessment of host genetic variation on this phase of the infection. In Exp. 1 larvae accumulated in the livers of both C57BL/6j and BALB/c mice up to and including days 4-5 p.i. and then declined in both strains until day 9. Loss of larvae from the livers corresponded to arrival in the lungs and maximum accumulation on day 7 p.i. but recovery was considerably higher in $\mathrm{C} 57 \mathrm{BL} / 6 \mathrm{j}$ mice. It was concluded that day 7 recoveries gave the best indication of relative resistance/susceptibility to this parasite. In Exp. $2 \mathrm{~A} / \mathrm{J}, \mathrm{BALB} / \mathrm{c}, \mathrm{CBA} / \mathrm{Ca}, \mathrm{C} 57 \mathrm{BL} / 6 \mathrm{j}, \mathrm{C} 3 \mathrm{H} / \mathrm{HeN}, \mathrm{DBA} / 2, \mathrm{NIH}, \mathrm{SJL}$, and SWR mice were compared. C57BL/6j mice were identified as the most susceptible strain and $\mathrm{CBA} / \mathrm{Ca}$ mice as having the most contrasting phenotype, but with a similar kinetic pattern of migration. Finally, in Exp. 3, a strong positive correlation between the size of the inoculum and the mean worm recovery from the lungs was found in CBA/Ca and C57BL/6j mice, but the difference between these strains was highly consistent, $66 \cdot 6-80 \%$, regardless of the initial dose. These results demonstrate that, using our protocols for infection and recovery, between-experiment variation in $\mathrm{A}$. suum worm burdens is minimal, and that C57BL/6j mice are highly susceptible to infection compared to other strains. The mechanistic basis of this susceptibility in relation to the resistance of other strains is unknown, but the possibilities are reviewed.

Key words: Ascaris suum, Ascaris lumbricoides, mouse model, pigs, C57BL/6j, CBA/Ca, susceptibility, lungs, migration.

\section{INTRODUCTION}

Ascaris lumbricoides and A. suum are important parasites of humans and pigs respectively (O'Lorcain and Holland, 2000; Crompton, 2001). Their importance lies primarily in the heavy adult worm burdens that can accumulate in predisposed subjects living in communities where transmission is intense (Holland and Boes, 2002) and similarly in pigs where husbandry procedures permit transmission (Boes et al. 1998). Additionally, both parasites also cause liver and lung pathology when hosts are exposed to large numbers of infective eggs simultaneously, as a result of the synchronized migration of the larvae and temporary development in these organs on their migration route to the intestine. Pulmonary symptoms can be pronounced and even life threatening and are known as Loeffler's syndrome (Loeffler, 1932, 1956; Vogel and Minning, 1942).

Recently there has been renewed interest in the consequences of Ascaris larvae migrating through the

* Corresponding author: School of Natural Sciences, Department of Zoology, Trinity College, University of Dublin, Dublin 2, Ireland. Tel: +353 (1) 608 2194. Fax: +353(1)6778094.E-mail: lewispr@tcd.ie lungs in the context of the hygiene and related hypotheses (Cooper et al. 2000), and yet to-date very little is known about this phase of the parasite's life-cycle. Recent comparative studies on larval migration in pigs and mice have led to the suggestion that the mouse is a suitable model for exploring susceptibility in the early phase of $A$. suum infection (Murrell et al. 1997; Slotved et al. 1998) as the pattern of migration of the larvae is analogous. The recovery of larvae from mice has been shown to be higher when compared to other abnormal hosts such as rabbits, guinea-pigs and rats (Douvres and Tromba, 1971) and even pigs (Roepstorff et al. 1997). Relative host size and parasite size contribute to this higher recovery.

Like most other helminths Ascaris show an aggregated distribution in their hosts, and although recent studies have suggested a genetic basis to this phenomenon in humans (Williams-Blangero et al. 2002), behavioural, immunological and physiological factors are also thought to play key roles in causing predisposition (McSharry et al. 1999; Holland and Boes, 2002). The mechanistic basis of predisposition is not understood, but it may well be initiated during the early phase of infection. Indeed, Cooper et al. (2000) have suggested that pulmonary migrations of 
Ascaris larvae could create a highly polarized Th2 immune environment in the lung mucosa, and genetic variation in the host response at this level may influence the course of subsequent infection. Since mice are known to vary in their susceptibility to infection with Ascaris (Mitchell et al. 1976), they provide a convenient model for investigating the basis of variation in establishment and in the success of subsequent migration of larvae through the liver and lungs.

In this paper we report a series of experiments which explore the kinetics of early Ascaris infection in the small intestine, liver and lungs of inbred mice. We first optimized the methodology for worm recovery and, on the basis of earlier publications, chose two inbred mouse strains to confirm that pronounced differences in migration kinetics exist. We then tested the hypothesis that genetic factors play a key role in influencing the success of larval migration, by comparing migration in a panel of mouse strains, incorporating some of those used by earlier workers but focusing particularly on the strains that have been highlighted for the mouse genome projects. We also explored the degree to which migration success is influenced by the dose of larvae administered and hence the size of the migrating population of worms. Finally, the effects of strain, day post-infection and dose on worm length were assessed.

MATERIALS AND METHODS

\section{Experimental animals}

Male mice were purchased from Harlan, UK at 7 weeks of age and were 8 weeks old upon commencement of the initial infections. Seventy inbred mice (35 C57BL/6j and $35 \mathrm{BALB} / \mathrm{c}$ ) were purchased for Exp. 1. A further 180 inbred mice were acquired for Exp. 2, 20 each of; A/J, BALB/c, C57BL/6j, $\mathrm{C} 3 \mathrm{H} / \mathrm{HeN}, \mathrm{CBA} / \mathrm{Ca}, \mathrm{DBA} / 2$, NIH, SJL and SWR. Finally, in Exp. 3, 180 inbred mice, 90 of the selected susceptible strain C57BL/6j and 90 of the resistant strain $\mathrm{CBA} / \mathrm{Ca}$, were purchased. Animals were maintained in conditions that were standard and constant; approximately $22^{\circ} \mathrm{C}$ with a daily $12 \mathrm{~h}$ photoperiodicity. Water and pelleted commercial food were provided ad libitum. The cages were cleaned and sawdust changed regularly.

\section{Parasite}

Approximately 4000000 embryonated ova (batch no.: 8/2002) were provided by the Danish Centre for Experimental Parasitology (CEP), Copenhagen. The eggs were removed from an isolate of $A$. suum taken from pigs at a Danish slaughterhouse. The eggs were isolated from the worm uteri and cultured to infectivity in $0 \cdot 1 \mathrm{M} \mathrm{H}_{2} \mathrm{SO}_{4}$ for 3 months (in the dark at room temperature), conditions that have been shown to result in fully infective eggs (Oksanen et al. 1990).
Upon arrival in Dublin, these eggs were diluted to a concentration of $25 \mathrm{eggs} / \mu \mathrm{l}$ as this is the maximum egg density which does not inhibit the infectivity or viability of the eggs (Eriksen, 1990). Prior to inoculation, the doses were adjusted to the desired number of fully embryonated eggs.

\section{Experimental protocol}

The preparation of a typical inoculum of 500 eggs was prepared as follows. A standard dose of $100 \mu \mathrm{l}$ of egg suspension containing 5 eggs $\mu \mathrm{l}^{-1}$ was drawn from the stock egg suspension during magnetic stirring and gentle agitation by hand, into an automatic pipette fitted with a disposable tip. The contents were emptied into a $1.5 \mathrm{ml}$ Eppendorf tube. Higher egg doses were concentrated from the stock solution. Using a modified version of the method described by Slotved (1997) we evaluated the number of eggs in the prepared dosages and thus standardized the number of eggs in an inoculation dose. Briefly, the numbers of eggs containing larvae were counted under $\times 10$ magnification in $10 \times 10 \mu \mathrm{l}$ samples. The procedure was repeated 6 times and the tip of the automatic pipette used to dispense the eggs onto the slide was changed between each replication.

Mice were inoculated by gastric intubation between 8 and 10 a.m. The inoculation apparatus, which consisted of a $1 \mathrm{ml}$ graduated syringe containing the relevant dose with a gastric intubation needle, was guided into the stomach of the mouse and the appropriate dose was administered.

All mice were euthanased by cervical dislocation on the relevant post-mortem day. Mice were weighed pre-infection and on the day of post-mortem and in experiments 2 and 3 the weight of the liver and spleen was also recorded at post-mortem. The days on which larvae were recovered post-inoculation (p.i.) were days 3-9 (Exp. 1), days 6-9 (Exp. 2) and days 6-8 (Exp. 3).

Experiment 1. Mice were inoculated with 500 fully embryonated eggs by stomach intubation. Five mice were euthanased from each strain daily between days 3 and 9 post-inoculation. At post-mortem examination, the liver and the lung were removed for examination of larval burdens. This experiment provided data on the larval kinetics in the lungs and liver of these reference strains, which were believed to represent susceptible and resistant phenotypes. It also tested the modified Baermann method that had been developed for recovering larvae from macerated organs.

Experiment 2. Mice were inoculated with 500 embryonated eggs and 5 mice of each strain were euthanased daily between days 6 and 9 p.i. to determine larval burdens in the lungs. This experiment was designed to determine which 2 strains showed 
the most contrasting phenotypes with respect to susceptibility to A. suum larval infection. Susceptibility was assessed by the number of larvae recovered from the lungs. The experiment provided additional data on the consistency of the recovery protocol.

Experiment 3. Mice were inoculated with 1 of 4 single inoculum doses $(3000,1000,500$, or 100$)$ and 2 doses of 1000 ova administered over consecutive days in split doses $(250 \times 4$ and $500 \times 2)$. Groups of 5 mice were euthansed daily between days 6 and 8 p.i. The lungs were examined for larval burden. The objective of this experiment was to determine the influence of dose on the larval burden not only within a strain, but also between different strains, and to provide further data on the consistency of the protocol.

\section{The modified Baermann method (adapted from Eriksen, 1981)}

The modified Baermann apparatus was constructed using a mesh comprising a double layer of gauze (sterile absorbent type 13 light gauze [Boots Pharmacy]) fixed with a rubber band to a plastic tube (20 ml universal sample tube) from which the bottom was removed. This gauze-tube apparatus was suspended in a $50 \mathrm{ml}$ centrifuge tube with the gauzed end inserted into the tube. Saline $(0.9 \%)$ was added to this apparatus up to the $35 \mathrm{ml}$ mark. The centrifuge tube apparatus was suspended up to the $15 \mathrm{ml}$ mark in a water bath at $37 \cdot 5^{\circ} \mathrm{C}$, overnight prior to post-mortem. On the day of post-mortem the liver and lungs were macerated using fine scissors. The macerated organ was placed on top of the gauze net and warm $0.9 \%$ saline was added to ensure the sample was completely submerged. The centrifugetube apparatus was capped to prevent loss of liquid. This apparatus was kept at a constant $37 \cdot 5{ }^{\circ} \mathrm{C}$ in the water bath for $24 \mathrm{~h}$.

\section{Recovery of worms}

After overnight incubation the gauze mesh was removed from the centrifuge tubes and the tubes capped. These were then centrifuged for $5 \mathrm{~min}$ at $1000 \mathrm{~g}$. After centrifugation, the solution (supernatant) was extracted carefully, so as not to disturb the pellet, using an aspirator, until $10 \mathrm{ml}$ of solution with the pellet beneath remained. Formalin $(6 \%)$ was added and the solution was allowed to settle for $24 \mathrm{~h}$ at room temperature. This was then re-centrifuged for $5 \mathrm{~min}$ at $1000 \mathrm{~g}$ and reduced to $4 \mathrm{ml}$.

\section{Larval counts}

The remaining $4 \mathrm{ml}$ solution and pellet in the centrifuge tube was agitated using a vortex mixer. Five $200 \mu \mathrm{l}$ samples were then placed on a slide and the number of larvae were counted with a phase-contrast stereomicroscope at $\times 40$ magnification. The sum of the larvae counted in the 5 aliquots was then calculated using the appropriate calculations.

\section{Measurement of larval length}

During larval counts, larvae that were suitable for measuring were photographed using an Olympus camedia digital camera C5050 (5.0 megapixels with a F1.6 lens) at $\times 10$ magnification. These images were transferred to a computer (OS windows XP) and each individual was measured using the programme Image J $1.32 \mathrm{j}$. Larvae were measured by drawing a segmented line through the centre of the body from the anterior to the posterior end.

\section{Statistical analysis}

Larval recovery was assessed for normality visually and statistically. Values for means, standard deviations, ranges, skewness, kurtosis and standard errors were obtained for each subset of data. The influence of the various factors on larval burden and size were analysed by means of analysis of variance (ANOVA in SPSS 12.0.1), using 1-, 2- or 3-way ANOVAs depending on the number of explanatory factors and covariates involved. Full-factorial models were simplified by step-wise deletion of non-significant terms beginning with the 3-way interactions, and minimum sufficient models in which only significant terms remained were used to assess significance of fitted factors. In some cases least squares difference (LSD) post-hoc tests were applied to tease out the major sources of variation within factors. Continuous variables were examined by Spearman's rank correlation. Statistical analysis was carried out at a confidence limit of $95 \%(\alpha=0 \cdot 05)$.

\section{RESULTS}

\section{Mean larval burdens}

In Exp. 1 the numbers of larvae in the liver and lungs were observed on days 3-9 post-inoculation (p.i.) to identify the migration patterns of larvae through these organs (Fig. 1). Larval counts in the liver changed significantly with time in both mouse strains (2-way ANOVA with time and strain as factors, model $R^{2}=0 \cdot 75$, main effect of time $F_{6,54}=22 \cdot 0$, $P<0 \cdot 0001)$, and the strains differed significantly (main effect of strain $F_{1,54}=4 \cdot 5, P=0.038$ and 2-way interaction strain $*$ time $F_{6,54}=2 \cdot 3, P=0 \cdot 05$ ), larvae moving into the livers of $\mathrm{C} 57 \mathrm{BL} / 6 \mathrm{j}$ at a faster rate than in $\mathrm{BALB} / \mathrm{c}$. This was particularly evident by the larger worm burdens on day 3 p.i., although by day 4 p.i. the mean larval burdens were similar in both strains. After this, worm burdens stabilized in $\mathrm{BALB} / \mathrm{c}$ mice but continued to rise in $\mathrm{C} 57 \mathrm{BL} / 6 \mathrm{j}$ mice until day 5 , after which there was a steady decline to 


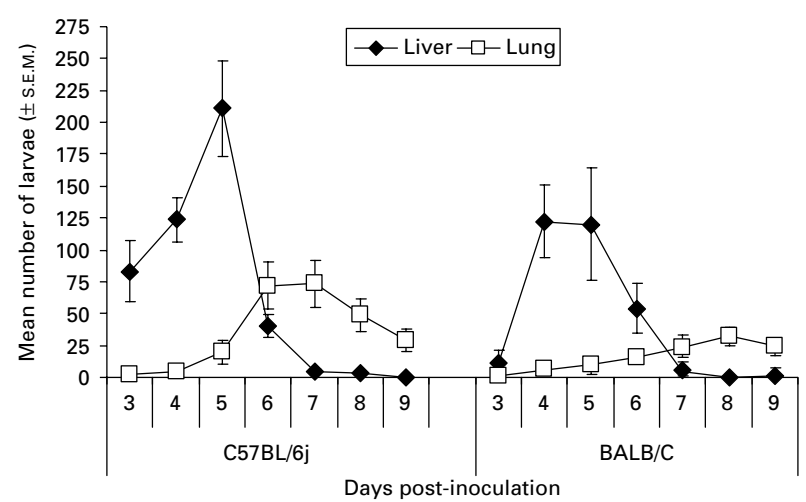

Fig. 1. The mean number ( \pm s.E.M. $)$ of larvae recovered from the liver $(\checkmark)$ and the lung $(\square)$ in the susceptible $(\mathrm{C} 57 \mathrm{BL} / 6 \mathrm{j})$ and resistant $(\mathrm{BALB} / \mathrm{c})$ strains of mice on days $3-9$ p.i.

zero by day 9 p.i. in both strains (Fig. 1). The highest percentage recovery of the initial inoculum from the liver was $42 \cdot 2 \%$ in $\mathrm{C} 57 \mathrm{BL} / 6 \mathrm{j}$ on day 5 and $24.4 \%$ in $\mathrm{BALB} / \mathrm{c}$ on day 4.

The reduction in larval numbers in the liver coincided with the movement of larvae into the lungs on days 5-6 p.i. in both strains, and again this was reflected in significant changes in worm burdens with time (2-way ANOVA with strain and time as factors on lung worm burdens, model $R^{2}=0 \cdot 743$, main effect of time $\left.=F_{6,54}=8 \cdot 99, P<0 \cdot 0001\right)$. Overall, in both strains, there was a reduction in the number of larvae that successfully migrated to this organ in comparison to the numbers observed in the liver. By day 7 p.i., in $\mathrm{C} 57 \mathrm{BL} / 6 \mathrm{j}$, the majority of larvae were found in the lungs ( $14.7 \%$ of the inoculated dose). However, in respect of lung worm burdens there was a more marked difference between strains compared with liver counts (main effect of strain, $F_{1,54}=15 \cdot 8$, $P=0.0002$ and 2-way interaction strain $*$ time, $F_{6,54}=3 \cdot 58, P=0.005$ ). The greatest difference in the mean number of larvae recovered between the 2 strains was observed in the lungs $(\geqslant 67 \cdot 4 \%)$ on days 6 and 7 p.i. This is in contrast to the liver, which although displaying higher larval numbers showed less of a percentage difference in worm burdens on the days of peak recovery $(=43 \cdot 1 \%)$.

Six standard inoculation dosages of 500 eggs, chosen randomly, were examined prior to inoculation and gave a mean of 493.5 eggs $( \pm 3 \cdot 8)$ confirming the consistency of administered doses and showing that variation in the administered dose was unlikely to make a significant contribution to the observed variance in larval burdens.

\section{Strain differences}

Exp. 2 employed 9 strains of mice to compare their relative susceptibilities to infection with $A$. suum and to identify for future work, 2 strains that show the greatest contrast of resistance/susceptibility

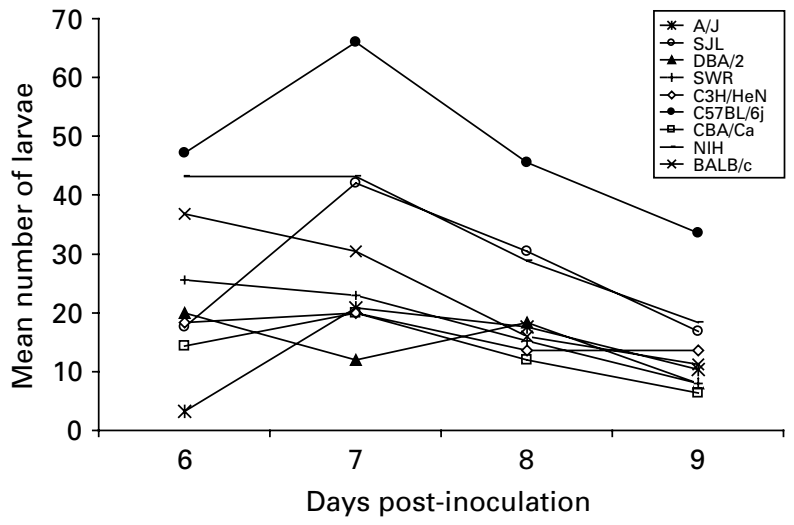

Fig. 2. The mean number of larvae recovered from the lungs of 9 strains of mice on days $6-9$ p.i.

phenotype. The results are illustrated in Fig. 2 and show that for the reference strain $\mathrm{C} 57 \mathrm{BL} / 6 \mathrm{j}$ the mean number of larvae recovered and the migratory kinetics in the lungs on the 4 days examined were indistinguishable from those observed in Exp. 1. Across all strains worm burdens in the lungs changed significantly with time (2-way ANOVA with day and strain as explanatory factors, model $R^{2}=0 \cdot 61$, on the lung worm burdens, main effect of time, $F_{3,140}=14 \cdot 2$, $P \leqslant 0 \cdot 0001)$. As can be seen, the strains differed markedly in lung worm burdens throughout the period after infection (main effect of strain, $\left.F_{8,140}=17 \cdot 95, P \leqslant 0 \cdot 0001\right)$ but in general the pattern of changes in worm burdens with time did not differ significantly between strains (2-way interaction strain $*$ day $F_{24,176}=1 \cdot 517, P=$ N.S.), indicating consistency in the daily changes in worm burdens across mouse strains.

Post-hoc analysis identified those strains that differed significantly from each other. Thus, C57BL/6j differed significantly from all strains $(P \leqslant 0 \cdot 0001)$, with a mean larval burden of $66 \pm 8 \cdot 2(13 \cdot 2 \%$ of the administered inoculum recovered) on day 7 p.i. and are classified as the most susceptible strain. NIH, $\mathrm{SJL}$ and BALB/c mice, in which mean larval recoveries on day 7 ranged from 6 to $8 \%$, were significantly different from the 6 other strains, but not each other and are classified as intermediate susceptible strains. Finally A/J, C $3 \mathrm{H} / \mathrm{HeN}, \mathrm{CBA} /$ $\mathrm{Ca}, \mathrm{DBA} / 2$ and SWR, with worm burdens of less than $4 \%$, were not significantly different from each other but were significantly different from the other 2 groupings of intermediate susceptible and most susceptible (C57BL/6j), and are classified as resistant strains.

The greatest significant difference between strains (1-way ANOVA, confined to day 7 p.i., model $R^{2}=0 \cdot 61$, effect of strain, $\left.F_{8,34}=6 \cdot 8, P \leqslant 0 \cdot 0001\right)$ was observed on day 7 post-inoculation. This was also the day on which larvae accumulated to their maximum number in the lungs of the susceptible C57BL/6j mouse strain and the day on which this strain differed 
most markedly from the other strains. In 4 of the other strains, A/J, SJL, C3H/HeN and CBA/Ca worm burdens were also maximum on day 7 p.i., but not in $\mathrm{DBA} / 2$, SWR, NIH and $\mathrm{BALB} / \mathrm{c}$ mice. However, while in the latter strains worm recoveries were higher on day 6 , the difference between day 6 and day 7 counts was marginal.

The most marked overall difference was detected between $\mathrm{CBA} / \mathrm{Ca}$ and $\mathrm{C} 57 \mathrm{BL} / 6 \mathrm{j}$ (post-hoc test, $P=0 \cdot 000014)$, which despite the large difference in numbers of larvae recovered on each day, showed the same pattern of worm migration during the 4-day period (Fig. 2). The percentage difference in the mean number of larvae recovered from these 2 strains on the 4 days was also highly consistent ranging between 69 and $81 \%$. These recoveries contrast with the next most susceptible strain $\mathrm{C} 3 \mathrm{H} / \mathrm{HeN}$, the recovery of larvae from this strain being as consistent but with a lower percentage difference relative to C57BL/6j mice, 59-5-70.2\%. It must be noted that although DBA/2 mice appeared to be most resistant on day 7 p.i., accumulation of larvae occurred in the lungs on day 6 in this strain (Fig. 2). While A/J mice appeared to be highly resistant overall, there was a great deal of variation among individuals of this strain with a range of values on the day of peak recovery, from 0 to 40 larvae, contrasting with $\mathrm{CBA} / \mathrm{Ca}$ mice where the range of individual burdens was considerably more restricted (12-28 larvae).

As an additional precaution, an analysis was undertaken that took host body weight into consideration. Lung worm burdens were expressed as worms per gram of body weight (total worm burden from the lungs/mouse body weight; Table 1). Once again, the two-way ANOVA for the number of larvae recovered per gram of mouse body weight (model $\left.R^{2}=0 \cdot 59\right)$ revealed that the main effects of strain $\left(F_{8,140}=15 \cdot 0, \quad P \leqslant 0 \cdot 0001\right)$ and day $\left(F_{3,140}=14 \cdot 7\right.$, $P \leqslant 0 \cdot 0001)$ were highly significant explanatory factors for variation in larval burdens but in this case there was a marginally significant 2 -way interaction (strain $*$ day, $F_{24, \mathbf{1 4 0}}=1 \cdot 61, P \leqslant 0 \cdot 0468$ ). Interestingly, post-hoc analysis confirmed a comparable outcome to that cited above, with the relative ranking of mouse strains identical to that concluded on the basis of total worm burdens.

Finally, we assessed whether there were any significant differences in body weight between the mouse strains that might have confounded our analysis. The analysis revealed that the body weight of mouse strains varied significantly (2-way ANOVA, with strains and time as factors, on mouse weight, main effect of strain, $F_{8,140}=31.9$, $P \leqslant 0 \cdot 0001$ ), but not with time (day p.i.) and the 2 -way interaction between strain and day was not significant. Post-hoc analysis showed that 1 strain differed from all of the other strains; NIH mice were significantly heavier than all the other strains. Thus no relationship emerged that related host body weight to parasite burdens. For example BALB/c and NIH differed significantly in their body weight (Table 1) but not in the number of worms recovered per gram of body weight.

\section{The influence of dose on mean larval numbers}

The outcome of this experiment was analysed in 2 stages. First we analysed the dose response with single-pulse infections (100, 500, 1000 and 3000 larvae) administered to $\mathrm{C} 57 \mathrm{BL} / 6 \mathrm{j}$ and $\mathrm{CBA} / \mathrm{Ca}$ mice. As previously, there was a highly significant difference between the strains with time and dose taken into consideration (Fig. 3, 2-way ANOVA with strain $*$ time + dose as a covariate, model $R^{2}=0 \cdot 70$, main effect of strain $\left.F_{1,114}=30 \cdot 4, P<0 \cdot 001\right)$ but no significant interaction between strain and time. Because this experiment was restricted to just 3 days, and given the variation in recovery within subgroups of mice, overall worm burdens did not vary significantly with time (main effect of days $F_{2,114}=2 \cdot 4$, $P=$ N.S.). Nevertheless, as in both of the earlier experiments, the arithmetic mean worm burdens were highest on day 7 in $\mathrm{C} 57 \mathrm{BL} / 6 \mathrm{j}$ mice at all 4 doses. This was also the case in the lower 3 doses $(100,500$ and 1000 eggs) of CBA/Ca mice. There was an exception in CBA/Ca mice inoculated with 3000 eggs with the highest level of infection observed on day 6 .

There was a highly significant positive relationship between the dose of eggs administered and the worm burdens in the combined data set with strain and time taken into account (effect of dose $F_{1,114}=242 \cdot 1$, $\beta=0 \cdot 129, t=15 \cdot 6, P<0 \cdot 001)$ and post-hoc within each of the 2 strains when analysed separately (1-way ANOVAs with time as a factor day, main effect of dose, $\beta=0 \cdot 179, \quad R_{\mathrm{adj}}{ }^{2}=0 \cdot 848, \quad t=13.98 \quad \mathrm{C} 57 \mathrm{BL} / 6 \mathrm{j}$ mice and $\beta=0 \cdot 079, R_{\mathrm{adj}}{ }^{2}=0 \cdot 903, t=18 \cdot 68$, for CBA/ $\mathrm{CA}$ and $P<0 \cdot 0001$ in both cases) suggesting a very strong correlation between the administered dose size and the number of larvae recovered within each strain. However, unexpectedly, the percentage of the initial inoculum recovered, on the day of peak accumulation, increased with increasing dose size (Fig. 4). In $\mathrm{C} 57 \mathrm{BL} / 6 \mathrm{j}$ mice percentage recoveries rose from $7 \cdot 2 \%$ in mice given just 100 larvae to $21 \cdot 6 \%$ in those given 3000. Similarly in CBA/Ca mice there was also a trend of increasing worm recoveries from $2 \cdot 4 \%$ to $7 \cdot 2 \%$. However, the percentage difference in worm burdens between C57BL/6j and CBA/Ca mice was independent of doses of larvae administered, and ranged from 66.6 to $78.9 \%$ (Fig. 4; Spearman's rank correlation on day 7 p.i., $R_{S}=0 \cdot 179, n=20$, $P=$ N.S.), implying that as the percentage of the initial inoculum recovered increased within the strains, the percentage difference between the 2 strains was constant and did not vary with increasing dose size and thus the disparity between the strains was maintained across the range of infection doses examined. 
Table 1. The mean number of larvae recovered from the lungs per gram of mouse body weight ( \pm s.E.) from each strain: C57BL/6j, NIH, SJL, $\mathrm{BALB} / \mathrm{c}, \mathrm{SWR}, \mathrm{C} 3 \mathrm{H} / \mathrm{HeN}, \mathrm{CBA} / \mathrm{Ca} \mathrm{A} / \mathrm{J}$ and DBA/2 on days 6-9 p.i. and the mean percentage of the initial inoculation dose of 500 Ascaris suum eggs recovered in this organ

(Also shown is the mean weight of the group on the day of post-mortem.)

\begin{tabular}{|c|c|c|c|c|c|}
\hline Strain & $n$ & Days p.i. & Post-mortem weight & Lung & Percentage \\
\hline $\mathrm{C} 57 \mathrm{BL} / 6 \mathrm{j}$ & $\begin{array}{l}5 \\
5 \\
5 \\
5\end{array}$ & $\begin{array}{l}6 \\
7 \\
8 \\
9\end{array}$ & $\begin{array}{l}24 \cdot 86( \pm 0 \cdot 81) \\
23 \cdot 27( \pm 0 \cdot 37) \\
22 \cdot 98( \pm 0 \cdot 38) \\
22 \cdot 74( \pm 0 \cdot 43)\end{array}$ & $\begin{array}{l}1 \cdot 90( \pm 0 \cdot 40) \\
2 \cdot 83( \pm 0 \cdot 33) \\
1 \cdot 98( \pm 0 \cdot 46) \\
1.48( \pm 0 \cdot 16)\end{array}$ & $\begin{array}{r}9 \cdot 4 \\
13 \cdot 2 \\
9 \cdot 1 \\
6 \cdot 7\end{array}$ \\
\hline $\mathrm{NIH}$ & $\begin{array}{l}5 \\
5 \\
5 \\
5\end{array}$ & $\begin{array}{l}6 \\
7 \\
8 \\
9\end{array}$ & $\begin{array}{l}26 \cdot 94( \pm 0.58) \\
25 \cdot 58( \pm 0 \cdot 63) \\
27 \cdot 51( \pm 0 \cdot 82) \\
25 \cdot 61( \pm 0 \cdot 63)\end{array}$ & $\begin{array}{l}1 \cdot 63( \pm 0 \cdot 32) \\
1 \cdot 71( \pm 0 \cdot 34) \\
1 \cdot 08( \pm 0 \cdot 28) \\
0 \cdot 71( \pm 0 \cdot 11)\end{array}$ & $\begin{array}{l}8 \cdot 6 \\
8 \cdot 6 \\
5 \cdot 8 \\
3 \cdot 7\end{array}$ \\
\hline SJL & $\begin{array}{l}5 \\
4 \\
5 \\
5\end{array}$ & $\begin{array}{l}6 \\
7 \\
8 \\
9\end{array}$ & $\begin{array}{l}23 \cdot 35( \pm 0 \cdot 28) \\
23 \cdot 55( \pm 0 \cdot 69) \\
21 \cdot 65( \pm 0 \cdot 30) \\
22 \cdot 33( \pm 0 \cdot 44)\end{array}$ & $\begin{array}{l}0.75( \pm 0 \cdot 09) \\
1.78( \pm 0 \cdot 43) \\
1 \cdot 40( \pm 0 \cdot 21) \\
0.74( \pm 0 \cdot 14)\end{array}$ & $\begin{array}{l}3 \cdot 5 \\
8 \cdot 4 \\
6 \cdot 1 \\
3 \cdot 4\end{array}$ \\
\hline $\mathrm{BALB} / \mathrm{c}$ & $\begin{array}{l}5 \\
5 \\
5 \\
5\end{array}$ & $\begin{array}{l}6 \\
7 \\
8 \\
9\end{array}$ & $\begin{array}{l}21 \cdot 45( \pm 0 \cdot 50) \\
21 \cdot 19( \pm 0 \cdot 41) \\
20 \cdot 81( \pm 0 \cdot 85) \\
21 \cdot 66( \pm 1 \cdot 09)\end{array}$ & $\begin{array}{l}1 \cdot 71( \pm 0 \cdot 30) \\
1 \cdot 44( \pm 0 \cdot 26) \\
0 \cdot 75( \pm 0 \cdot 26) \\
0 \cdot 51( \pm 0 \cdot 09)\end{array}$ & $\begin{array}{l}7 \cdot 4 \\
6 \cdot 1 \\
3 \cdot 2 \\
2 \cdot 2\end{array}$ \\
\hline SWR & $\begin{array}{l}5 \\
4 \\
5 \\
5\end{array}$ & $\begin{array}{l}6 \\
7 \\
8 \\
9\end{array}$ & $\begin{array}{l}20 \cdot 99( \pm 0 \cdot 57) \\
19 \cdot 36( \pm 1 \cdot 11) \\
19 \cdot 44( \pm 1 \cdot 03) \\
18 \cdot 84( \pm 0 \cdot 93)\end{array}$ & $\begin{array}{l}1 \cdot 22( \pm 0 \cdot 10) \\
1 \cdot 20( \pm 0 \cdot 36) \\
0 \cdot 78( \pm 0 \cdot 13) \\
0 \cdot 41( \pm 0 \cdot 11)\end{array}$ & $\begin{array}{l}5 \cdot 1 \\
4 \cdot 6 \\
3 \cdot 0 \\
1 \cdot 6\end{array}$ \\
\hline $\mathrm{C} 3 \mathrm{H} / \mathrm{HeN}$ & $\begin{array}{l}5 \\
5 \\
5 \\
5\end{array}$ & $\begin{array}{l}6 \\
7 \\
8 \\
9\end{array}$ & $\begin{array}{l}23 \cdot 11( \pm 0 \cdot 28) \\
23 \cdot 40( \pm 0 \cdot 66) \\
21 \cdot 94( \pm 1 \cdot 21) \\
22 \cdot 77( \pm 0 \cdot 33)\end{array}$ & $\begin{array}{l}0 \cdot 81( \pm 0 \cdot 44) \\
0.87( \pm 0 \cdot 15) \\
0 \cdot 62( \pm 0 \cdot 17) \\
0.59( \pm 0 \cdot 14)\end{array}$ & $\begin{array}{l}3 \cdot 7 \\
4 \\
2 \cdot 7 \\
2 \cdot 7\end{array}$ \\
\hline $\mathrm{CBA} / \mathrm{Ca}$ & $\begin{array}{l}5 \\
5 \\
5 \\
5\end{array}$ & $\begin{array}{l}6 \\
7 \\
8 \\
9\end{array}$ & $\begin{array}{l}19 \cdot 62( \pm 0 \cdot 46) \\
21 \cdot 01( \pm 0 \cdot 49) \\
21 \cdot 24( \pm 0 \cdot 73) \\
19 \cdot 97( \pm 0 \cdot 33)\end{array}$ & $\begin{array}{l}0 \cdot 74( \pm 0 \cdot 22) \\
0.95( \pm 0 \cdot 12) \\
0 \cdot 58( \pm 0 \cdot 10) \\
0 \cdot 32( \pm 0 \cdot 08)\end{array}$ & $\begin{array}{l}2 \cdot 9 \\
4 \\
2 \cdot 4 \\
1 \cdot 3\end{array}$ \\
\hline $\mathrm{A} / \mathrm{J}$ & $\begin{array}{l}5 \\
5 \\
5 \\
5\end{array}$ & $\begin{array}{l}6 \\
7 \\
8 \\
9\end{array}$ & $\begin{array}{l}21 \cdot 68( \pm 0 \cdot 17) \\
21 \cdot 86( \pm 0 \cdot 17) \\
21 \cdot 07( \pm 0 \cdot 12) \\
21 \cdot 46( \pm 0 \cdot 05)\end{array}$ & $\begin{array}{l}0 \cdot 14( \pm 0 \cdot 07) \\
0 \cdot 92( \pm 0 \cdot 29) \\
0 \cdot 83( \pm 0 \cdot 11) \\
0 \cdot 47( \pm 0 \cdot 18)\end{array}$ & $\begin{array}{l}0 \cdot 6 \\
4 \cdot 2 \\
3 \cdot 5 \\
2 \cdot 1\end{array}$ \\
\hline $\mathrm{DBA} / 2$ & $\begin{array}{l}4 \\
5 \\
5 \\
4\end{array}$ & $\begin{array}{l}6 \\
7 \\
8 \\
9\end{array}$ & $\begin{array}{l}21 \cdot 08( \pm 0 \cdot 77) \\
21 \cdot 20( \pm 0 \cdot 91) \\
19 \cdot 25( \pm 1 \cdot 14) \\
22 \cdot 27( \pm 1 \cdot 33)\end{array}$ & $\begin{array}{l}0.95( \pm 0.23) \\
0.57( \pm 0.21) \\
0.94( \pm 0.19) \\
0.35( \pm 0.07)\end{array}$ & $\begin{array}{l}4 \\
2 \cdot 4 \\
3 \cdot 7 \\
1 \cdot 6\end{array}$ \\
\hline
\end{tabular}

The second stage of the analysis was confined to mice that had received 1000 eggs but either as a single dose, as 2 doses or as 4 doses. The results are illustrated in Fig. 5. These show that under the split dose regime, the difference between the strains was preserved (3-way ANOVA, with infection regime, strain and time as factors on worm burdens in the lungs, model $R^{2}=0 \cdot 547$, main effect of strain, $F_{1,70}=44 \cdot 7$, $P<0.001)$, the 2 strains carrying significantly different worm burdens on different days after infection ( 2 way interaction strain $*$ time, $F_{2,70}=3 \cdot 1, P=0 \cdot 05$ ), especially on day 6 rather than on day 7 . Only in the single pulse infection was the peak accumulation of larvae in the lungs evident on day 7 in $\mathrm{C} 57 \mathrm{BL} / 6 \mathrm{j}$ mice. Nevertheless, the percentage difference between strains was $69 \%(500 \times 2$ dose group $)$ and $76 \%$
$(250 \times 4)$ on day 6 , which is a similar percentage difference when compared to the single inoculum groups. The 3 infection regimes clearly produced significantly different patterns of worm recovery overall (main effect of regime, $F_{1,70}=12 \cdot 7, P<0 \cdot 001$ ) and on different days after infection (2-way interaction regime $*$ time, $\left.F_{4,70}=3 \cdot 17, \quad P=0 \cdot 019\right)$. The 2 strains of mice behaved differently (2-way interaction strain $*$ regime, $\quad F_{2,70}=3.93, \quad P=0.02$ and 3 -way interaction, $F_{4,70}=2 \cdot 8, P=0 \cdot 032$ ) with worm burdens being more consistent over the 3 -day period in $\mathrm{CBA} / \mathrm{Ca}$ mice compared to $\mathrm{C} 57 \mathrm{BL} / 6 \mathrm{j}$, where worm burdens were considerably higher on day 7 (single dose of 1000 eggs) or day 6 (the other two regimes) compared to day 8 . There was also a highly significant main effect of time $\left(F_{2,70}=6 \cdot 8, P=0 \cdot 002\right)$, 
(A)



(C)



(B)

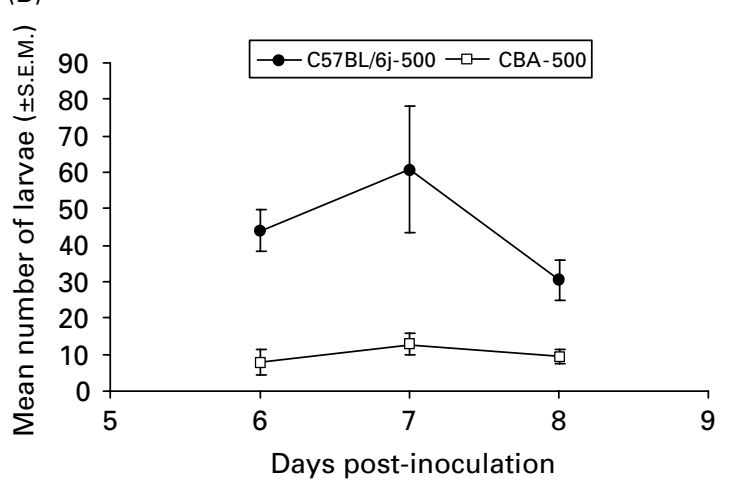

(D)

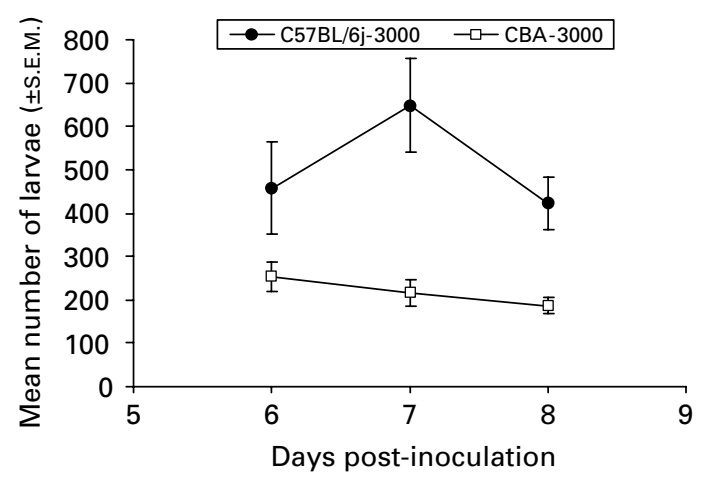

Fig. 3. The mean number ( \pm s.E.M.) of larvae recovered from the lungs of both the C57BL/6j and CBA/Ca mice administered 1 of 4 single-pulse inoculations; (A) 100, (B) 500, (C) 1000 and (D) 3000 Ascaris suum ova on days 6-8 p.i.

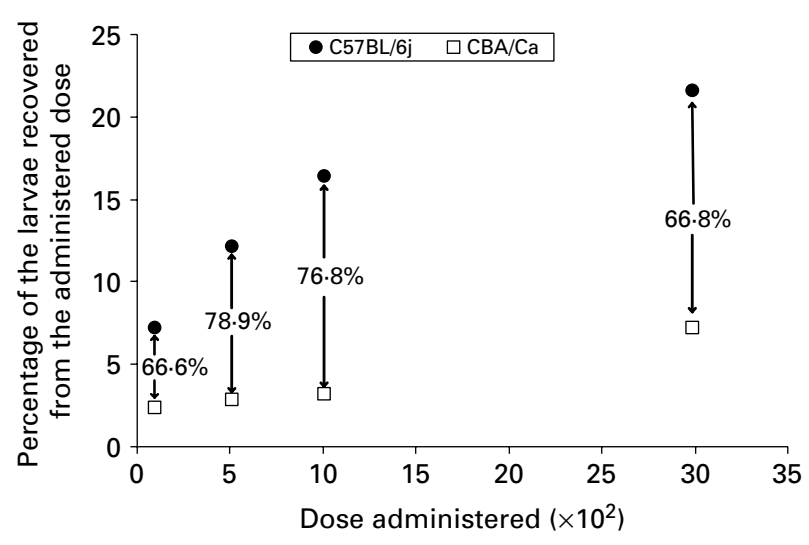

Fig. 4. The percentage of larvae recovered on day 7 p.i. after the initial inoculation doses of 100, 500, 1000 and 3000 from the lungs of the mouse strain C $57 \mathrm{BL} / 6 \mathrm{j}$ and the strain $\mathrm{CBA} / \mathrm{Ca}(\square)$ and the percentage difference between the 2 strains. (Percentage difference calculated as: Total number of larvae recovered from $\mathrm{C} 57 \mathrm{BL} /$ $6 \mathrm{j}-$ Total number of larvae recovered from $\mathrm{CBA} / \mathrm{Ca} /$ Total number of recovered from $\mathrm{C} 57 \mathrm{BL} / 6 \mathrm{j}$ ).

with worm burdens generally declining across the 3-day period of the experiment.

The effect of strain, time and dose on growth of larvae

An analysis of the body length of larvae and the factors resulting in variation in growth were investigated. The 3 explanatory factors used to determine variation in larval length were: strain of mouse, dose of larvae and day after infection. The infection protocol comprised animals given single pulse infection and split dose infections and these were analysed separately.

\section{Effect of increasing doses of eggs on the growth of larvae}

The mean sizes of larvae recovered from the lungs of mice exposed to $100,500,1000$ or 3000 eggs, reflecting growth over the 3 -day period examined, are illustrated in Fig. 6. There were insufficient numbers of viable larvae recovered from the 100-dose group to assess growth confidently.

As expected, there was a highly significant increase in larval length during the three days that larvae were observed (initially 3-way ANOVA, with strain and time as factors and dose as covariate, full factorial model reduced to a minimum sufficient model (adjusted model $R^{2}=0 \cdot 329$ ) comprising only time, dose and the 2-way interaction between time and dose, main effect of time, $F_{2,185}=27 \cdot 95, P \leqslant 0 \cdot 001$ ). The minimum individual larval length was observed on day $6(474 \mu \mathrm{m})$ and the maximum length observed was on day $8(1687 \mu \mathrm{m})$. Growth was influenced by dose of eggs administered (main effect of dose, $\left.F_{1,185}=17 \cdot 7, P \leqslant 0 \cdot 001\right)$ reflecting density-dependent constraints on growth (Fig. 6), with faster growth in 
(A)

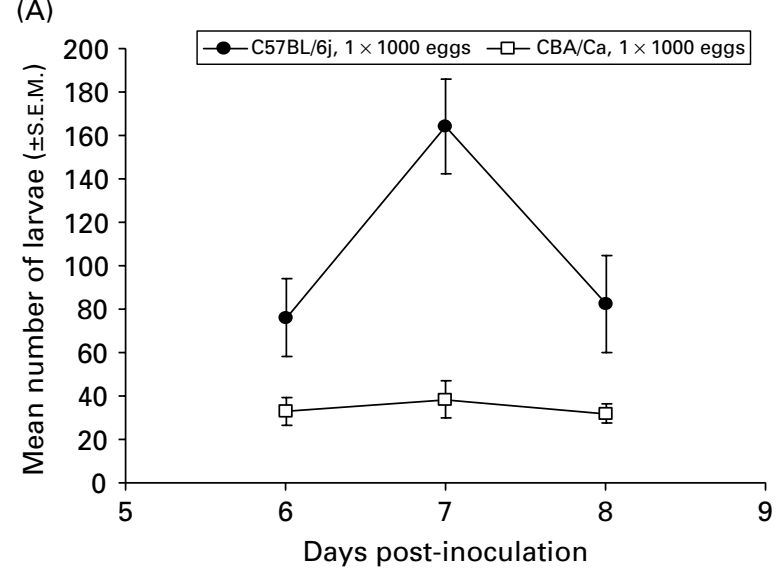

(B)

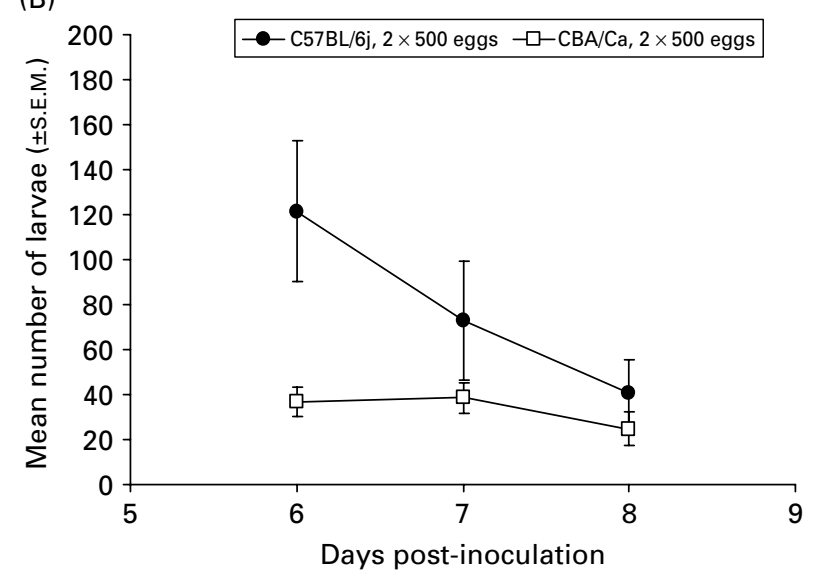

(C)

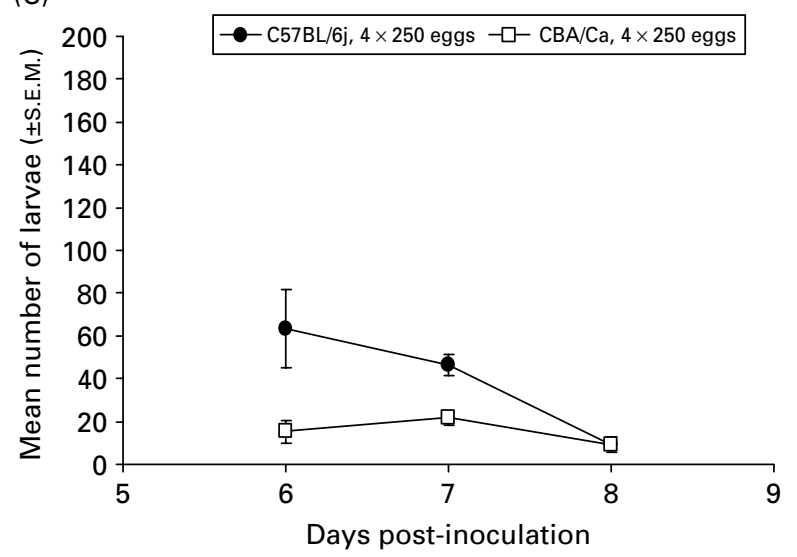

Fig. 5. The mean number ( \pm s.E.) of larvae recovered from the lungs of both $\mathrm{C} 57 \mathrm{BL} / 6 \mathrm{j}$ and $\mathrm{CBA} / \mathrm{Ca}$ mice administered with a single inoculum of 1000 ova (A) and those inoculated with a trickle dose of either $250 \times 4$ (B) or $500 \times 2$ (C) of Ascaris suum ova during the infection period (day $6-8$ p.i.).

mice exposed to the lowest inoculum, and slowest in the mice given 3000 eggs. This is most evident by pattern of growth between day 6 and 7 after infection (Fig. 6). Larval length in the animals exposed to 1000 eggs was an intermediate length but was less consistent than in the other groups, larvae failing to increase in length between days 7 and 8

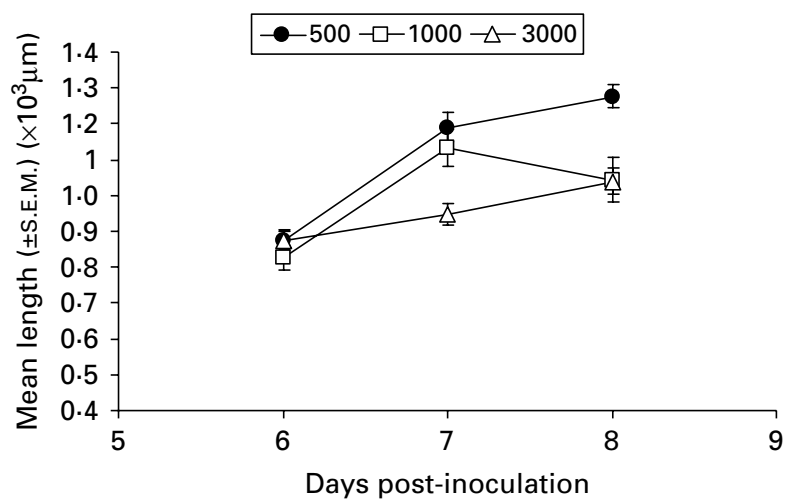

Fig. 6. Mean body length of larvae ( \pm s.E.) recovered from the lungs of mice administered with a single inoculum of 3000, 1000 or 500 Ascaris suum ova during the 3 -day period (6-8) under investigation.

(2-way interaction between time $*$ dose, $F_{2,185}=7 \cdot 9$, $P=0 \cdot 001)$. There was no difference between the strains in the growth of larvae (Full factorial model, main effect of strain $F_{1,179}=2 \cdot 9, P=$ N.S. and all interactions involving strain were not significant).

\section{Effect of a split-dose of 1000 eggs on the growth of larvae}

The second component of this experiment was assessment of growth under a single or 2 split-dose protocols. In all 3 cases the total number of eggs administered was 1000 , but 1 group received this dose over a period of 2 days and another over 4 days, simulating to a degree natural exposure to the parasite. As above, there was no significant difference between the strains of mice, and no significant interactions with dose or time involving strain. Strain and all its interactions were therefore removed from the model. There was strong evidence for growth of the larvae with time after infection (main effect of time on worm length $F_{2,146}=9 \cdot 5, P<0.001$ ), and growth was clearly affected by whether larvae were given in one dose or split into several doses (main effect of dose regimen $\left.F_{2,146}=3 \cdot 4, \quad P=0 \cdot 037\right)$. Initially on day 6 , assuming that only the larvae from the first inoculum would have arrived in the lungs, a dose-dependent effect was apparent (Table 2), the longest larvae being from the mice infected with $4 \times 250$ larvae, but thereafter there was little growth in this group. Best growth was observed in mice exposed to $2 \times 500$ eggs. An LSD post-hoc analysis between the dose groups showed that the doses of 1000 and $500 \times 2$ were most different and that larval length differed significantly between day 6 and the 2 other post-inoculation days. Clearly the overall picture was not as clear-cut as in the mice exposed to single pulse infections because it was confounded by the arrival of larvae from the eggs given on days 2,3 and 4 . 
Table 2. Mean larval length \pm s.E. $(n)$ on days 6,7 , and 8 post-inoculation in the lungs of the susceptible $(\mathrm{C} 57 \mathrm{BL} / 6 \mathrm{j})$ and resistant $(\mathrm{CBA} / \mathrm{Ca})$ strains of mice inoculated with either $1000,500 \times 4$ or $250 \times 2$ infective eggs of Ascaris suum

\begin{tabular}{llcc}
\hline \hline & & \multicolumn{1}{c}{ C57BL/6j } & CBA/Ca \\
Dose & Day p.i. & Length $(\mu \mathrm{m}) \pm$ s.E. $(n)$ & Length $(\mu \mathrm{m}) \pm$ s.E. $(n)$ \\
\hline 1000 & 6 & $838 \pm 27(10)$ & $818 \pm 64(12)$ \\
& 7 & $1133 \pm 66(10)$ & $1130 \pm 92(7)$ \\
$500 \times 2$ & 8 & $1088 \pm 71(12)$ & $940 \pm 135(5)$ \\
& 6 & $918 \pm 64(17)$ & $1092 \pm 66(9)$ \\
& 7 & $1203 \pm 64(8)$ & $1090 \pm 97(8)$ \\
$250 \times 4$ & 8 & $1240 \pm 51(9)$ & $1244 \pm 158(6)$ \\
& 7 & $1093 \pm 91(12)$ & $909 \pm 10(2)$ \\
& 8 & $1086 \pm 70(12)$ & $872 \pm 66(3)$ \\
\hline \hline
\end{tabular}

\section{DISCUSSION}

This study provides strong evidence that inbred strains of mice differ markedly in their susceptibility to early $A$. suum infection as measured by larval burdens in the lungs on day 7 post-infection. Although there was some disparity in the movement of larvae into the lungs of a number of strains (BALB/c, DBA/2), this variation can be accounted for in part by the smaller number of larvae recovered in these strains compared with the most susceptible $\mathrm{C} 57 \mathrm{BL} / 6 \mathrm{j}$ mice and the associated variation generating apparent changes in the timing of migration. However, this does not impact on the significance of the analysis. The maximum accumulation in the susceptible strain $\mathrm{C} 57 \mathrm{BL} / 6 \mathrm{j}$ was consistently observed on day 7 and as such this day post-infection is suggested as the best day for assessing differences in susceptibility between strains of mice. However, it is recommended that larval migration is observed on days 6-8 also, to ensure that peaks of accumulation either side of day 7 are not missed when comparing new strains of mice. C57BL/6j were uniquely the most susceptible strain, exhibiting consistency in larval burden in the lung over 3 experiments and maintaining their susceptibility at lower and higher doses of infective ova. Other inbred strains fell into 2 categories - the intermediate susceptible group and the resistant group. $\mathrm{CBA} / \mathrm{Ca}$ were selected as the most resistant strain based upon the similarity in the kinetics of their migration, with the susceptible strain, over the 4-day period of assessment and the highly significant difference in larval numbers between the two strains on day 7. Crucially, the differences in the larval burden were not attributable to host body weight (a possible confounding factor), since heavier mice were not more susceptible to increased larval burdens.

Earlier work by Mitchell et al. (1976) demonstrated variability in susceptibility to Ascaris in the lungs of inbred strains of mice but variations in infectivity of batches of eggs and doses resulted in lack of comparability between groups. For example, the average recovery of larvae from the lungs of C $57 \mathrm{BL} / 6 \mathrm{j}$ varied from $14.5 \%$ to $0.73 \%$ in 2 separate experiments with varying dosage administered. Johnstone, Leventhal and Soulsby (1978) reported large variation in the number of larvae recovered from the lungs of $\mathrm{C} 57 \mathrm{BL} / 6 \mathrm{j}$ mice as a result of using 2 different protocols for the recovery of larvae $(8.2 \%$ to $5 \cdot 3 \%$ between 2 procedures). However, the larval burdens observed here in the $\mathrm{BALB} / \mathrm{c}$ mice are comparable to the recoveries achieved by Slotved et al. (1997) with higher percentages of the initial inoculum recovered $(4 \cdot 3 \%$ (Slotved et al. 1997) compared to $7 \cdot 3 \%$ and $6.6 \%$ in the current experiments). It is pertinent that their recoveries of worms do not correspond to the observations of Mitchell et al. (1976) who concluded that BALB/c were the most resistant strain, with a mean recovery of $7( \pm 1)$ or $0.04 \%$ of the administered dose. The methodology employed in the present study with resultant consistency between separate experiments in worm recoveries from uniformly treated mice, and consistent between-strain variation as well as a significantly higher proportion of larvae being recovered compared to previous studies (Johnstone et al. 1978; Slotved et al. 1997, 1998), all represent marked improvements on earlier published studies.

The dose did not influence the relative susceptibility of the 2 strains. Therefore it can be assumed that there are underlying intrinsic host factors resulting in the observed phenotypes, as the difference in the migrating larval population between the 2 strains remained constant irrespective of the initial dose size. Within strains there was a correlation between the inoculum size and the mean number of larvae recovered. There was also a positive densitydependent relationship between dose and the percentage of the initial inoculum that was recovered, as 
percentage larval recovery increased with increasing inoculum size. This pattern was observed in both the susceptible and resistant strains of mice, and is consistent with observations by Johnstone et al. (1978) on $A$. suum in the lungs of $\mathrm{C} 57 \mathrm{BL} / 6$ mice, but in other host-parasite systems it is more usual to observe a reduction in percentage establishment with increasing parasite density (see for example Norozian-Amiri and Behnke, 1993 and below for further discussion).

In most cases, the protective immune response against helminths residing in the intestinal tract is a Th2 response. When immune responses are effective at eliminating tissue stages of these parasites, there is abundant evidence that this can be orchestrated by the Th1 arm of the immune system (Mulcahy et al. 2005). A common genetic variant involved in Th-2 immune signalling, STAT6 (signal transducers and activators of transcription), a mediator of Th2 responses in helminth infections, predicts increased resistance to adult $A$. lumbricoides infections (Peisong et al. 2004). Such Th2 responses result in increased mucosal eosinophil and mast cell activity, mucus hypersecretion and muscle hyperactivity in the gut and they represent conditions favourable for the expulsion of helminths. In contrast the opposing effect of STAT6 has been observed in relation to susceptibility to larval stage of Taenia crassiceps. STAT6 deficiency is known to impair IL-4 signalling and thus the ability to generate a Th2 response. Wild type BALB/c mice developed a strong Th-2 like response and remained susceptible to $T$. crassiceps larval stages. However, similarly infected STAT6 ${ }^{-/-}$mice mounted a strong Th-1 like response and controlled infection (Rodriguez-Sosa et al. 2002). This variation in Th1 and Th2 responses may play a role in variations in susceptibility to early-stage $A$. suum infection between mouse strains. Individuals that naturally upregulate the Th2 response during larval migration may cause suppression of a Th1 immune response and thus show an increased susceptibility to infection. Furthermore there is the possibility that increased administration of infective ova, and thus successfully migrating larvae, resulted in greater preferential stimulation of the Th2 response, aiding parasite evasion of host defences or increasing their survival by further down-regulation of the Th1 parasite specific responses, especially in the susceptible mouse strain.

Larval burdens that established in the lungs as the result of an inoculation divided over time (split doses) exhibited lower numbers compared to an equivalent single dose and this was particularly pronounced in the $\mathrm{C} 57 \mathrm{BL} / 6 \mathrm{j}$ mice. The underlying reasons may relate to a differential immune response to gradual inoculation or the influence of stress due to increased handling. Some evidence for a differential immune response is provided by the reduced larval burden in the 1000 ova dose administered over 4 days in contrast to the dose administered over a period of 2 days and the single pulse administration of 1000 eggs. Mice have been shown to be resistant to an Ascaris challenge infection shortly after primary inoculation or when repeatedly inoculated at short intervals (Mitchell et al. 1976; Eriksen, 1981). Increased cellular response in the liver of reinoculated abnormal hosts resulted in increased encapsulation of larvae (Sprent and Chen, 1949; Taffs, 1965). Thus mice exposed to $A$. suum ova over a period of time would be expected to have an increased reaction to larvae in the early stages of migration and this would be observed as fewer larvae successfully penetrating the lungs during the latter stages. Overall, it was concluded that the 500 or 1000 single doses represented the optimum dose range for further experiments exploring the basis of the differential susceptibility between the 2 selected strains without generating unnecessarily heavy worm burdens in the mice and placing undue stress upon the mice.

Larval length was not influenced by strain suggesting that the environment within both strains was equally suitable for the development of the worms and that the observed differences in relative susceptibility are unlikely to be attributable to differences in larval fitness but rather to innate barriers to migration or differences in the early phase of the host response to the parasite. Johnstone et al. (1978) demonstrated that in immunised mice, resistance affected the stage to which $A$. suum larvae develop and also the length to which they grow. An expected observation was the increase in larval length over the three days post-inoculation examined (Douvres and Tromba, 1971; Johnstone et al. 1978). There was a correlation between dose administered and larval lengths, lower doses resulting in an increase in the average larval length, especially on day 7 p.i. The mean larval length was consistently higher among worms recovered from mice exposed to 500 eggs, indicating that dose exerts an important influence on the growth of worms. The observation that as dose increased, more worms were successful in completing the migration to the lungs, even though they did not grow as well as when fewer were present, suggests that there may be an active host component that underlies the difference between strains. One possibility is that with increasing worm burden damage (e.g. lesions in the gut mucosa, liver, blood vessels) to host tissues provided greater opportunities for onward migration for relatively more worms, and at the same time stimulated greater innate resistance which impeded worm development, and hence resulted in slower growth.

Perhaps the most intriguing question to emerge from these experiments is why C57BL/6 mice are so much more susceptible than other strains of mice. The C57BL/6 strain has also been described as susceptible to Brachylaima cribbi, Plasmodium 
berghei, Strongyloides ratti, and Toxoplasma gondii (Dawkins et al. 1980; Butcher, Palethorpe and Grove, 2002; de Souza and Riley, 2002; Lee and Kasper, 2004). It is known that the ancestors of C57BL/6 (and also C57BL10 and C58BL) diverged from the lineage that gave rise to other laboratory strains early on during domestication and selection for traits for laboratory work (Beck et al. 2000). These 3 strains form a clade of their own. Equally interesting are recent data that indicate that C57BL/6j mice do not express the gene for intelectin-2, which is believed to play an important role in resistance to GI nematodes such as Trichinella spiralis (Pemberton et al. 2004). The authors suggest that the presence of intelectin-2 in resistant BALB/c mice, its absence from susceptible C57BL/10 strain and the kinetics of its up-regulation during $T$. spiralis infection are all consistent with this novel lectin serving a protective role in the innate immune response to parasite infection.

At this stage, we cannot be certain about the mechanism underlying the greater susceptibility of C57BL/6j mice to infection with Ascaris but it is likely to be genetic and to consist of innate or molecular/structural/physiological differences between the strains with one set of alleles enabling Ascaris to migrate in large numbers and the other impeding this migration. The early variation in the larval burdens observed in the liver between C57BL/6j and BALB/c in experiment one and the differences in the timing of the accumulation in this organ suggest that larvae may be lost early in infection. One possibility is a difference in larval penetration of the large intestine between the two strains (Slotved et al. 1998). Mouse strains possibly vary in the activation signals that they provide for embryonated eggs to hatch, and eggs may pass through the intestine without hatching. Equally it is conceivable that more larvae are entrapped in the livers of the resistant strain, or that their onward migration from the liver is less successful than in the susceptible strain. Finally it is possible that on arriving in the lungs more larvae fail to establish in resistant mice, or are expelled possibly through the bronchial airways by coughing.

We are now confident that our experimental procedures can yield consistency in Ascaris larval lung burdens between 2 inbred strains of mice. This model system will enable exploration of the mechanisms underlying the observed susceptibility and resistance to early Ascaris infection, an aspect of the host-parasite relationship that has proved very difficult to investigate in human and porcine hosts for financial, logistical and ethical reasons. Furthermore, successful identification of the gene or genes underlying the different (resistance/susceptibility) phenotypes of $\mathrm{CBA} / \mathrm{Ca}$ and $\mathrm{C} 57 \mathrm{BL} / 6 \mathrm{j}$ might provide an avenue for finding homologous genes in pigs and humans in the future.
We thank the Irish Research Council for Science Engineering and Technology and the EMBARK initiative for funding the research, and Professor Lis Eriksen in the Royal Veterinary College in Denmark for provision of $A$. suum infective eggs and her help and support. We would also like to thank Alison Boyce, Clare Hamilton and Brian Murphy for their hard work in the laboratory.

\section{REFERENCES}

Beck, J. A., Lloyd, S., Hafezparaast, M., LennonPierce, M., Eppig, J. T., Festing, M. F. W. and Fisher, E. M. C. (2000). Genealogies of mouse inbred strains. Nature Genetics 24, 23-25.

Boes, J., Medley, G. F., Eriksen, L., Roepstorff, A. and Nansen, P. (1998). Distribution of Ascaris suum in experimentally and naturally infected pigs and comparison with Ascaris lumbricoides infections in humans. Parasitology 177, 589-596.

Butcher, A. R., Palethorpe, H. M. and Grove, D. I. (2002). The susceptibility of inbred mice to infection with Brachylaima cribbi (Digenea: Brachylaimidae). Parasitology International 51, 109-115.

Cooper, P. J., Chico, M. E., Sandoval, C., Espinel, I., Guevara, A., Kennedy, M. W., Urban, J. F. Jr., Griffin, G. E. and Nutman, T. B. (2000). Human infection with Ascaris lumbricoides is associated with a polarised cytokine response. Fournal of Infectious Diseases 182, 1207-1213.

Crompton, D. W. T. (2001). Ascaris and ascariasis. Advances in Parasitology 48, 285-375.

Dawkins, H. J. S., Grove, D. I., Dunsmore, J. D. and Mitchell, G. F. (1980). Strongyloides ratti: susceptibility to infection and resistance to reinfection in inbred strains of mice as assessed by excretion of larvae. International Fournal for Parasitology 10, 125-129.

de Souza, J. B. and Riley, E. M. (2002). Cerebral malaria: the contribution of studies in animal models to our understanding of immunopathogenesis. Microbes and Infection 4, 291-300.

Douvres, F. W. and Tromba, F. G. (1971). Comparative development of Ascaris suum in rabbits, guinea pigs, mice and swine in 11 days. Proceedings of the Helminthological Society of Washington 38, 246-252.

Eriksen, L. (1981). Host-parasite relations of Ascaris suum infection in pigs and mice. Ph.D. thesis. Institute of Internal Medicine, Royal Veterinary and Agriculture University, Copenhagen.

Eriksen, L. (1990). Ascaris suum influence of egg density and in vitro development from embyonated egg to infective egg. Acta Veterinaria Scandinavica 31, 489-491.

Holland, C. V. and Boes, J. (2002). Distributions and predisposition: people and pigs. In The Geohelminths: Ascaris, Trichuris and Hookworm (ed. Holland, C. V. and Kennedy, M. W.), pp. 1-24. Kluwer Academic Publishers, Boston/Dordrecht/London.

Johnstone, C., Leventhal, R. and Soulsby, E. J. L. (1978). The spin method for recovering tissue larvae and its use in evaluating C57BL/6 mice as a model for the study of resistance to infection with Ascaris suum. Fournal of Parasitology 64, 1015-1020.

Lee, Y. H. and Kasper, L. H. (2004). Immune responses of different mouse strains after challenge with 
equivalent lethal doses of Toxoplasma gondii. Parasite 11, 89-97.

Loeffler, W. (1932). Zur Differentialdiagnose der Lungen-infiltrierungen. II Ueber flüchtige Succedanininfiltrate (mit Eosinophilie). Beiträge zur Klinik der Tuberkulose 79, 368-382.

Loeffler, W. (1956). Transient lung infiltrations with blood eosinophilia. International Archives of Allergy and Applied Immunity 8, 54-59.

McSharry, C., Xia, Y., Holland, C. V. and Kennedy, M. W. (1999). Natural immunity to Ascaris lumbricoides associated with immunoglobulin E antibody to ABA-1 allergen and inflammation indicators in children.

Infection and Immunity 67, 484-489.

Mitchell, G. E., Hogarth-Scott, R. S., Edwards, R. D., Lewers, H. M., Cousins, G. and Moore, T. (1976). Studies on immune response to parasite antigens in mice. 1. Ascaris suum larvae numbers and antiphosphorylcholine responses in infected mice of various strains and in hypothymic nu/nu mice.

International Archives of Allergy and Applied Immunity 52, 64-78.

Mulcahy, G., O’Neill, S., Fanning, J., McCarthy, E. and Sekiya, M. (2005). Tissue migration by parasitic helminths- an immunoevasive strategy? Trends in Parasitology 21, 273-277.

Murrell, K. D., Eriksen, L., Nansen, P., Slotved, H.-C. and Rasmussen, T. (1997). Ascaris suum: A revision of its early migratory path and implications for human ascariasis. Fournal of Parasitology 83, 255-260.

Norozian-Amiri, S. M. B. and Behnke, J. M. (1993). Density-dependent effects on establishment of Necator americanus and Ancylostoma ceylanicum. Fournal of Helminthology 67, 151-157.

Oksanen, A., Eriksen, L., Roepstorff, A., IIsoe, B., Nansen, P. and Lind, P. (1990). Embryonation and infectivity of Ascaris suum eggs. A comparison of eggs collected from worm uteri with eggs isolated from pig faeces. Acta Veterinaria Scandinavica 31, 393-398.

O'Lorcain, P. and Holland, C. V. (2000). The public health importance of Ascaris lumbricoides. Parasitology 121 (Suppl.) S51-S71.

Peisong, G., Mao, X.-Q., Enomoto, T., Feng, Z., Gloria-Bottini, F., Bottini, E., Shirakawa, T., Sun, D. and Hopkin, J. M. (2004). An asthma-associated genetic variant of STAT6 predicts low burden of Ascaris worm infection. Genes and Immunity 5, 58-62.

Pemberton, A. D., Knight, P. A., Gamble, J., Colledge, W. H., Lee, J.-K., Pierce, M. and Miller,
H. R. P. (2004). Innate BALB/c enteric responses to Trichinella spiralis: inducible expression of a novel goblet cell lectin, intelectin-2, and its natural deletion in C57BL/10 mice. Fournal of Immunology 173, 1894-1901.

Rodriguez-Sosa, M., David, J. R., Bojalil, R., Satoskar, A. R. and Terrazas, L. I. (2002). Cutting Edge: Susceptibility to the larval stage of the helminth parasite Taenia crassiceps is mediated by Th2 response induced via STAT6 signalling. Fournal of Immunology 168, 3135-3139.

Roepstorff, A., Eriksen, L., Slotved, H.-C. and Nansen, P. (1997). Experimental Ascaris suum infection in the pig: worm population kinetics following single inoculations with three doses of infective eggs. Parasitology 115, 443-452.

Slotved, H.-C. (1997). Methods for quantitative recovery of larval and adult stages of nematodes (Ascaris suum and Oesophagostomum dentatum) in pigs and mice. Ph.D. thesis. Danish Centre for Experimental Parasitology, Royal Veterinary and Agricultural University, Copenhagen.

Slotved, H.-C., Eriksen, L., Murrell, K. D. and Nansen, P. (1997). Comparison of methods for recovery of Ascaris suum larvae from tissues of mice. International Fournal for Parasitology 27, 1305-1310.

Slotved, H.-C., Eriksen, L., Murrell, K. D. and Nansen, P. (1998). Early Ascaris suum migration in mice as a model for pigs. Fournal of Parasitology 84, $16-18$.

Sprent, J. F. A. and Chen, H. H. (1949). Immunological studies in mice infected with the larvae of Ascaris lumbricoides. I. Criteria of immunity and immunizing effect of isolated worm tissues. Fournal of Infectious Disease 84, 111-124.

Taffs, L. F. (1965). Immunological studies on experimental infection of guinea pigs and rabbits with Ascaris suum Goeze, 1782. IV. The histopathology of the liver and lung. Fournal of Helminthology 39, 297-302.

Vogel, H. and Minning, W. (1942). Beiträge zur Klinik der Lungen-ascariasis und zur Frage der flüchtigen, eosinophilen Lungeninfiltrate. Beiträge zur Klinik der Tuberkulose 98, 620-654.

Williams-Blangero, S., Vandeberg, J. L., Subedi, J., Aivaliotis, M. J., Rai, D. R., Upadhayay, R. P., Jha, B. and Blangero, J. (2002). Genes on chromosomes 1 and 13 have significant effects on Ascaris infection. Proceedings of the National Academy of Sciences, USA 99, 5533-5538. 